Angelina do Carmo Lessa 1

\title{
Comparação da situação nutricional de crianças de baixa renda no segundo ano de vida, antes e após a implantação de programa de atenção primária à saúde
}

\author{
Comparison of nutritional status of low-income \\ children in the second year of life before \\ and after primary health care intervention
}

1 Escola Paulista de Medicina, Universidade Federal de São Paulo. Rua Marselhesa 630 São Paulo, $S P$ 04020-060, Brasil. lessa.pnut@epm.br devincenzi.pnut@epm.br dirsigulem.pnut@epm.br

\begin{abstract}
The so-called "Slum Project" or Projeto Favela at the São Paulo School of Medicine, Federal University in São Paulo, conducts numerous primary health care activities in low-income communities in the city of São Paulo to prevent and control nutritional deficiencies. The aim of this study was to compare the nutritional status of children from 12 to 26 months of age before and after preventive intervention. The study was conducted with two groups, before and after intervention, with 57 and 65 children, respectively. Anthropometric measures and hemoglobin values were obtained. In the before-intervention group, the prevalence of malnutrition as measured by weight for age and weight for height was $12.3 \%$ and $1.8 \%$ respectively, whereas there were no cases of malnutrition in the after-intervention group. There was a reduction in the prevalence of anemia, especially the severe form, from 33\% to 7\%. Mean hemoglobin increased from 10.1 to $11.0 \mathrm{~g} / \mathrm{dl}$, and the duration of breastfeeding was greater (> 180 days) after intervention.
\end{abstract}

Key words Primary Health Care; Child Nutrition Disorders; Anemia; Breast-Feeding

Resumo O Projeto Favela, da Escola Paulista de Medicina, Universidade Federal de São Paulo, realiza diversas atividades de educação e promoção das ações básicas de saúde em comunidades carentes do Município de São Paulo, visando principalmente a prevenção e controle de carências nutricionais. $O$ objetivo do estudo foi comparar a situação nutricional de crianças com idades entre 12 e 26 meses, antes e após a implantação de um conjunto de ações preventivas. Formaram-se dois grupos, pré e pós implantação, com 57 e 65 crianças respectivamente, que foram submetidas à avaliação antropométrica e dosagem de hemoglobina. O grupo exposto a todas as ações não apresentou nenhum caso de desnutrição (0\%) para os indicadores P/I e P/E, comparado a 12,3\% e 1,8\%, respectivamente, no grupo pré implantação. Houve redução da prevalência de anemia, sobretudo a de maior gravidade, que passou de 33\% para 7\%, com aumento do valor médio da hemoglobina (de 10,1 g/dl para 11,0 g/dl) além de maior porcentual de crianças com aleitamento materno total acima de 180 dias no grupo pós-implantação.

Palavras-chave Cuidados Primários de Saúde; Transtornos da Nutrição Infantil; Aleitamento Materno; Anemia 


\section{Introdução}

O estado nutricional da criança é fortemente influenciado pela condição sócio-econômica, sendo que fatores ligados às características familiares, condições de habitação e cuidados de saúde são também importantes (Fernandes et al., 1996).

Durante as últimas décadas, observou-se evolução favorável no perfil nutricional das crianças brasileiras. Entre os fatores apontados como expressivos na explicação estão a expansão da cobertura de saneamento e a ampliação do acesso aos serviços básicos de saúde. Porém, Yunes \& Monteiro (1995) observaram que a ausência de programas articulados de saúde e educação podia ser apontada como um dos entraves para maiores avanços.

Embora se tenha alcançado maior cobertura dos serviços públicos, ainda existem milhares de crianças que sobrevivem em condições ambientais extremamente desfavoravéis. O processo de urbanização contribui para o aumento da cobertura dos serviços públicos, mas por outro lado, as peculiaridades de tal processo nos países em desenvolvimento (Santos, 1982), aumentou a pressão sobre a estrutura física e social das cidades, especialmente em relação à educação, habitação, saneamento e saúde sem, contudo, contar com uma infra-estrutura básica para atender tal demanda. Como conseqüência, tem-se um aumento da população residente em favelas, que ocupam moradias precárias e com serviços escassos de água, saneamento e atenção à saúde (OMS, 1989).

Na cidade de São Paulo, cerca de $12 \%$ das crianças até 5 anos residem em favelas e embora tenha ocorrido melhoria nas condições de saneamento ambiental, as situações insalubres ainda persistem (Monteiro \& Nazário, 2000). É importante então, reconhecer os efeitos prejudiciais que esse ambiente exerce sobre as condições de saúde e nutrição das crianças que são as mais afetadas, uma vez que são as mais expostas ao ambiente peridomiciliar e especialmente vulneráveis frente aos riscos para a saúde que este ambiente proporciona, sendo de primordial importância a atenção à saúde para esse grupo (OMS, 1989).

A transmissão de informações e programas de saúde e educação acessíveis é importante como estratégia para que se possa atingir essa população marginalizada, permitindo que se desenvolvam conhecimentos, técnicas e comportamentos necessários para a promoção da saúde (UNICEF, 1998). Essas atividades podem ser maximizadas, quando centradas nas ações básicas de saúde, tais como: promoção do alei- tamento materno, orientação ao desmame e à terapia de reidratação oral, incentivo à imunização, diagnóstico precoce e tratamento de infecções respiratórias agudas, e vigilância do crescimento e desenvolvimento da criança (UNICEF, 1979).

A partir da década de 70, com o reconhecimento da necessidade de ampliação da cobertura do setor saúde, a atenção primaria à saúde surge como alternativa de ação do setor e, em 1978, com a realização da Conferência de Atenção Primaria à Saúde, em Alma Ata, essa passa a figurar como a principal estratégia da Organização Mundial da Saúde (OMS) para se elevar as condições de saúde das populações. Durante a conferência, destacou-se a necessidade de priorizar os grupos mais vulneráveis e desprivilegiados da sociedade, e da realização de pesquisas continuadas que possibilitassem a avaliação para identificação de problemas e eficácia dos serviços, permitindo conhecer melhor as necessidades, prioridades e respostas às intervenções (Klein et al., 1982).

Nesse sentido, este trabalho tem o objetivo de comparar a situação nutricional de crianças residentes em comunidades carentes, antes e após a implantação de um conjunto de ações para prevenção e controle de carências nutricionais no contexto da atenção primária à saúde, avaliando a viabilidade e contribuição dessa intervenção na saúde e nutrição infantil.

\section{Metodologia}

Para desenvolver um modelo de atenção primaria à saúde, em 1989 foi criado o Projeto Favela, coordenado por um grupo de docentes da Escola Paulista de Medicina, Universidade Federal de São Paulo (EPM/UNIFESP). Inicialmente, realizou-se o levantamento sócio-econômico e nutricional de moradores de favelas da região circunvizinha à Instituição. Das 402 crianças menores de 5 anos avaliadas, $29,8 \%$ apresentaram algum grau de desnutrição. Os primeiros anos se caracterizaram, sobretudo, por atividades de mapeamento, diagnóstico da população materno-infantil e recuperação dos casos de desnutrição energético protéica (DEP) (Priore et al., 1994).

A partir de 1995 foi iniciada também a avaliação da anemia ferropriva, encontrando-se prevalência de $63,1 \%$ nas crianças menores de 36 meses (Devincenzi, 1999), o que fez com que nos anos de 1996 e 1997 a atuação fosse maior no tratamento da carência de ferro. A partir de 1998 foram então estabelecidas as ações preventivas conjuntas tanto para DEP como para 
anemia, estabelecendo-se também o acompanhamento sistemático mensal para o primeiro ano de vida.

As atividades desenvolvidas articulam ações de educação e promoção em saúde, compreendendo desde o acompanhamento da gestação (com avaliação do estado nutricional materno), o estímulo ao aleitamento (com visitas semanais no primeiro mês de vida da criança, para apoiar a nutriz e transmitir técnicas corretas, passando a ser regulares próximas ao mês completo, até os 12 meses), a orientação para a introdução da alimentação complementar, a vigilância da situação vacinal e do crescimento e desenvolvimento, o controle da anemia ferropriva por meio de educação nutricional e profilaxia com ferro medicamentoso (na dose de $1 \mathrm{mg}$ Fe elementar por kg de peso/dia, desde o desmame até os 24 meses de idade). $\mathrm{O}$ atendimento à população, realizado na grande maioria por nutricionistas, se dá de forma individual, no domicílio, e em grupos, por meio de reuniões nos centros comunitários localizados dentro das favelas. Cada criança possui um prontuário, no qual são anotados todos os dados do atendimento, que são transferidos para o banco de dados em Epi Info 6.02.

Para elaboração deste trabalho foram utilizadas informações do banco de dados do Projeto Favela Para tanto, foram definidos dois grupos com idade entre 12 e 26 meses, contando o primeiro com 57 crianças que tinham dados completos, representando $50 \%$ do universo de crianças nessa faixa etária, identificadas em 1995, quando ainda não haviam sido estabelecidas efetivamente todas as rotinas atuais de intervenção, denominado grupo pré-intervenção (GI) e o segundo grupo, denominado pós-intervenção (GII) composto por 65 crianças com coleta completa de dados, representando $62 \%$ do universo de crianças nessa faixa etária, identificadas no ano de 2000, quando as rotinas de intervenção já estavam efetivamente implantadas. As crianças do grupo II receberam um número mediano de 12 visitas ao longo do acompanhamento pelo Projeto Favela.

Foram estudadas variáveis sócio-demográficas da mãe e da criança, e dados antropométricos e bioquímicos da criança. A idade foi considerada em meses completos. A idade materna foi considerada na data do parto e expressa em anos completos. A escolaridade materna foi expressa em anos de estudo completos à data da avaliação.

A avaliação nutricional tanto para as crianças do GI como do GII, realizou-se por meio da antropometria, utilizando os indicadores peso/idade, comprimento/idade e peso/compri- mento. Para a obtenção do peso utilizou-se balança eletrônica, com precisão de $100 \mathrm{~g}$ e o comprimento foi aferido com antropômetro de madeira, graduado em centímetros, seguindo recomendações de Jelliffe (1968), e o padrão de referência foi o do National Center for Health Statistics. Os resultados dos indicadores antropométricos são apresentados em escore Z. Por se tratar de um trabalho de intervenção que objetiva sobretudo diagnosticar déficits precocemente, além do corte em - $2 \mathrm{Z}$ que caracteriza a desnutrição, também se adotou na apresentação dos resultados, o ponto de corte que caracteriza risco nutricional - escore $\mathrm{Z}>-2 \mathrm{e} \leq-1$ (WHO, 1995).

Para diagnosticar a presença de anemia, nos dois grupos, avaliou-se o nível sérico de hemoglobina por meio do hemoglobinômetro digital portátil - "Hemocue". O sangue é colhido por meio de punção digital, utilizando-se de material descartável e o resultado é fornecido pelo aparelho em g/dl (Ribeiro, 2001; Rosenbilit et al., 1999). Utilizou-se o ponto de corte de $11,0 \mathrm{~g} / \mathrm{dl}$ para diagnóstico da anemia (OMS, 1968), e foram considerados casos de maior gravidade os valores inferiores a 9,5g/dl (Monteiro \& Szarfarc, 1987).

O aleitamento materno foi classificado em predominante - TAMEP (leite materno, chá e água) e aleitamento total - TAMT (tempo total que a criança recebeu leite materno) (WHO, 1991). No GI, as informações foram coletadas retrospectivamente, tendo as crianças mais de 12 meses de idade, e no GII as informações foram obtidas a partir do acompanhamento longitudinal durante o primeiro ano de vida. Para análise dessa variável, foram estabelecidas categorias com pontos de corte baseados nas medianas de aleitamento do GII, coletadas prospectivamente, calculando-se a freqüência de crianças dos dois grupos nas categorias. O peso ao nascer foi informado pela mãe e quando necessário conferido com o cartão da maternidade, sendo classificado como baixo peso ao nascer (BPN), crianças com peso igual ou inferior a $2.500 \mathrm{~g}$ (WHO, 1977).

Foram utilizados os programas Epi Info 6.02 e SigmaStat 2.0 para formação do banco de dados e análises estatísticas. Os testes empregados foram $t$ de Student, o teste do $x^{2} \mathrm{e}$, quando indicado, o Teste Exato de Fischer para avaliar independência ou associação entre os grupos de estudo. O nível para rejeição da hipótese de nulidade em todos os testes foi de 0.05 ou $5 \%$, assinalando-se com asterisco $(*)$ os valores estatisticamente significantes. O presente estudo foi submetido e aprovado pelo Comitê de Ética em Pesquisa da EPM/UNIFESP. 


\section{Resultados}

Fizeram parte do estudo 118 crianças, sendo 57 do GI e 65 do GII. As variáveis sociodemográficas da população estudada estão representadas na Tabela 1. Os grupos apresentaram-se semelhantes quanto à escolaridade materna, com mediana de 4 anos completos de estudo para ambos $(p=0,49)$. Quanto à situação conjugal, também não houve diferença estatística, sendo que $15,8 \%$ das mães do GI e $17,7 \%$ do GII viviam sem companheiro ( $p=0,77)$. Já a idade materna foi diferente entre os grupos, observando-se redução da mediana de 25 (GI) para 22 anos (GII) ( $p=0,007)$, e aumento na proporção de mães adolescentes (GI = 7,1\%, GII = 22,9\%; $p=0,01)$. A distribuição das crianças, segundo o sexo, não apresentou diferença significante $(\mathrm{p}=0,34)$, não havendo também, diferença em relação à mediana de idade $(\mathrm{GI}=19$ meses; GII =20 meses; $\mathrm{p}=0,54$ ).

O peso médio ao nascer foi semelhante entre os grupos, com 3.110 e 3.119 gramas para o GI e GII, respectivamente (Tabela 2). Entretanto, o percentual de BPN reduziu de $14,3 \%$ para

Tabela 1

Características sociodemográficas de mães e crianças. Projeto Favela, São Paulo, Brasil, 1995-2000.

\begin{tabular}{|c|c|c|c|c|c|}
\hline \multirow[t]{2}{*}{ Estratos } & \multicolumn{2}{|c|}{ GI1 } & \multicolumn{2}{|c|}{ GII2 } & \multirow[t]{2}{*}{ Valor de $p^{3}$} \\
\hline & $\mathrm{n}$ & $\%$ & $\mathrm{n}$ & $\%$ & \\
\hline \multicolumn{6}{|l|}{ Idade materna } \\
\hline$\leq 18$ anos & 4 & 7,1 & 14 & 22,9 & 0,01 \\
\hline$>18$ anos & 52 & 92,9 & 47 & 77,1 & \\
\hline \multicolumn{6}{|l|}{ Escolaridade materna } \\
\hline$\leq 4$ anos completos & 32 & 57,1 & 31 & 50,8 & 0,49 \\
\hline$>4$ anos completos & 24 & 42,9 & 30 & 49,2 & \\
\hline \multicolumn{6}{|l|}{ Situação conjugal } \\
\hline com companheiro & 48 & 84,2 & 51 & 82,3 & 0,77 \\
\hline sem companheiro & 9 & 15,8 & 11 & 17,7 & \\
\hline \multicolumn{6}{|l|}{ Sexo criança } \\
\hline Feminino & 25 & 43,9 & 27 & 58,5 & 0,34 \\
\hline Masculino & 32 & 56,1 & 38 & 41,5 & \\
\hline \multicolumn{6}{|l|}{$\begin{array}{l}\text { Idade criança } \\
\text { (em meses) }\end{array}$} \\
\hline $12-18$ & 22 & 38,6 & 31 & 47,7 & 0,31 \\
\hline $18-26$ & 35 & 61,4 & 34 & 52,3 & \\
\hline
\end{tabular}

GI = grupo pré-intervenção; GII = Grupo pós-intervenção.

1 No Gl 1 criança não tinha informação de idade e escolaridade maternas.

2 No Gll 4 crianças não tinham informação de idade e escolaridade

maternas e 3 da situação conjugal.

3 Teste do $\chi^{2}$.
$6,3 \%$, embora sem significância estatística $(\mathrm{p}=$ $0,14)$.

As informações sobre o aleitamento materno, constantes das Tabelas $3 a$ e $3 b$, indicam maior tempo de duração para o GII. O percentual de crianças que não recebeu leite materno foi significantemente superior no GI, 33\% contra $10 \%$ no GII ( $\mathrm{p}=0,002$ ) e também houve maior percentual de crianças do GII com TAMEP acima de 60 dias, com tendência estatística $(p=0,09)$. Em relação ao tempo de aleitamento total, o percentual de crianças que foram amamentadas por mais de 180 dias foi superior no GII, $44 \%$ contra $19,5 \%$ no GI ( $p=0,01)$.

A caracterização dos déficits antropométricos das crianças está representada na Tabela 4 . A prevalência de desnutrição, segundo o indicador peso/idade mostrou diferença significante entre os grupos, sendo que no GII nenhuma criança apresentou escore menor ou igual a -2 e no GI, 7 (12,3\%) apresentaram o desvio ( $p=0,015$ ). Para o indicador peso/comprimento, nenhum dos dois grupos apresentou crianças com desnutrição, mas o percentual de crianças em risco nutricional foi maior no GI $(\mathrm{p}=0,004) \operatorname{com} 21 \%(\mathrm{n}=13)$ do que no GII com $4,9 \%(n=3)$. O indicador comprimento/idade não apresentou diferença entre os grupos, tanto para desnutrição como para risco nutricional. Ressalta-se que nas crianças do GII abaixo de $-2 \mathrm{Z}$ para o indicador $A / I(n=6)$, o escore $Z$ médio de $\mathrm{P} / \mathrm{I}$ foi de $-1,49$ (risco nutricional) $\mathrm{e}$ para $\mathrm{P} / \mathrm{A}$ o valor do escore médio foi de $-0,16$.

Para os valores de hemoglobina (Tabela 5), não foi possível obter dados de 4 crianças do GII, totalizando 57 crianças em cada grupo. A concentração média de hemoglobina do GI foi $10,1 \mathrm{~g} / \mathrm{dl} \pm 1,4$, enquanto no GII foi $11,0 \mathrm{~g} / \mathrm{dl} \pm$ $1,2(\mathrm{p}<0,001)$. Na subdivisão dos grupos em faixas etárias maior e menor que 18 meses, de forma semelhante à análise global, a concentração de hemoglobina foi maior nas duas faixas de idade para o GII. A prevalência de anemia mostrou-se significantemente inferior no GII, que apresentou $50,9 \%$ de crianças com diagnóstico de anemia, enquanto no GI esse valor foi de $69,5 \%$ ( $p=0,043)$. A anemia de maior gravidade sofreu redução ainda maior, com o GI apresentando prevalência de 33,3\% contra $7 \%$ do GII $(p=0,0004)$.

\section{Discussão}

Inicialmente, é importante fazer algumas considerações acerca do desenho do estudo apresentado. Segundo Kirkwood et al. (1997), para avaliar intervenções deve-se considerar que o 
estudo randomizado, duplo-cego e controlado é aceito como padrão-ouro, dando maior credibilidade às inferências sobre os resultados. Porém, até pela questão ética que possa envolver um estudo, pode-se optar por outros desenhos que permitem confiabilidade desde que apresente adequado controle e plausibilidade biológica. A exemplo, tem-se a comparação pré versus pós-intervenção. A plausibilidade estará demonstrada à medida em que se observar redução nos fatores de risco associados, assim como na redução do próprio problema em questão. Deve-se observar alguns fatores que podem influenciar o resultado, como a tendência secular e variações na incidência ou prevalência em diferentes faixas etárias.

É importante então considerar que a idade da criança é um fator de risco nutricional. Dentro dessa perspectiva, cabe destacar que os grupos estudados apresentaram idades semelhantes sendo, portanto, homogêneos quanto ao risco para a ocorrência da DEP e da anemia (Sigulem, 1981).

A idade e a escolaridade materna, assim como a situação conjugal estão associadas com diversas condições e constituem variáveis de risco para a saúde e nutrição da criança (Caetano, 1992; Schmitz, 1999). No presente estudo, a escolaridade materna não se alterou no período pré e pós-intervenção, apresentando valores semelhantes entre os grupos. No que se refere à idade materna, houve redução significante da mediana e aumento na proporção de mães adolescentes no GII. Em relação à situação conjugal, o percentual de mães sem companheiro também foi semelhante entre os grupos.

Essas análises permitem observar que fatores sócio-demográficos como idade e sexo da criança, escolaridade materna e situação conjugal mostraram-se semelhantes para os dois grupos, à exceção da idade materna, que se apresentou desfavorável para o grupo de intervenção (GII).

O BPN, representa um importante fator de risco tanto para a anemia como para DEP. As reservas de ferro ao nascer são proporcionais ao peso da criança, portanto, o menor peso ao nascimento representa menores reservas do mineral, com conseqüente aumento de risco para a anemia. Em relação à DEP, também se observa maior risco para crianças com BPN, revelandose mais vulneráveis às infecções, além de maior risco de retardo do crescimento (Sigulem, 1981). Para a cidade de São Paulo, o porcentual de BPN situa-se em $8,7 \%$, sendo que no tercil mais pobre da população esse número atinge 9,7\% (Monteiro et al., 2000a). Neste estudo, o peso médio ao nascer foi semelhante en-
Tabela 2

Peso médio ao nascer e percentual de BPN do GI e Gll. Projeto Favela, São Paulo, Brasil, 1995-2000.

\begin{tabular}{lccc}
\hline Peso ao nascer (gramas) & GI & GII & Valor de p \\
\hline Média (DP) & $3.110(631)$ & $3.119(457)$ & 0,81 \\
$\% \leq 2500$ & 14,3 & 6,3 & 0,142 \\
$\%>2500$ & 86,7 & 92,7 & \\
\hline
\end{tabular}

BPN = baixo peso ao nascer; GI = grupo pré-intervenção;

GII = Grupo pós-intervenção.

1 Teste $t$ de Student.

2 Teste do $\chi^{2}$.

Tabela $3 a$

Freqüência de aleitamento materno predominante (TAMEP), segundo categorias de tempo no GI e GII. Projeto Favela, São Paulo, Brasil, 1995-2000.

\begin{tabular}{|c|c|c|c|c|c|}
\hline \multirow{2}{*}{$\begin{array}{l}\text { Aleitamento } \\
\text { predominante }\end{array}$} & \multicolumn{2}{|c|}{ GI } & \multicolumn{2}{|c|}{ GII2 } & \multirow[t]{2}{*}{ Valor de $p^{3}$} \\
\hline & $\mathrm{n}$ & $\%$ & $n$ & $\%$ & \\
\hline Não1 & 19 & 33,3 & 6 & 10,0 & 0,02 \\
\hline Até 60 dias & 20 & 35,1 & 26 & 43,3 & 0,36 \\
\hline Acima 60 dias & 18 & 31,6 & 28 & 46,7 & 0,09 \\
\hline Total & 57 & 100,0 & 60 & 100,0 & \\
\hline
\end{tabular}

GI = grupo pré-intervenção; GII = Grupo pós-intervenção.

1 Inclui crianças que não inciaram o aleitamento ou introduziram

outro leite,conjuntamente, logo após o nascimento.

2 Cinco crianças sem informação.

3 Teste do $\chi^{2}$.

Tabela 3b

Freqüência de aleitamento materno total (TAMT), segundo categorias de tempo no GI e GII. Projeto Favela, São Paulo, Brasil, 1995-2000.

\begin{tabular}{lrrrrr}
\hline $\begin{array}{l}\text { Tempo total de } \\
\text { aleitamento }\end{array}$ & Gl12 & & GII3 & Valor de $\mathbf{p}^{4}$ \\
\hline Até 120 dias & 28 & 68,3 & 22 & 44,0 & 0,01 \\
De 120 até 180 dias & 6 & 12,2 & 6 & 12,0 & 0,98 \\
Acima de 180 dias & 8 & 19,5 & 22 & 44,0 & 0,01 \\
Total & 42 & 100,0 & 50 & 100,0 & \\
\hline
\end{tabular}

GI = grupo pré-intervenção; GII = Grupo pós-intervenção.

1 não inclui crianças que não foram amamentadas, sendo 7 do Gl e 2 do Gll.

2 Oito crianças sem informação.

3 Treze crianças sem informação.

4 Teste do $\chi^{2}$ 
Tabela 4

Prevalência de desnutrição e de risco nutricional das crianças de Gl e Gll, segundo indicadores. Projeto Favela, São Paulo, Brasil, 1995-2000.

\begin{tabular}{lccc}
\hline Indicadores & GI & GII & Valor de $\mathbf{p}^{1}$ \\
\hline P/I & & & \\
Escore $\leq-2$ & 12,3 & 0 & $0,015^{\star}$ \\
Escore $>-2$ e $\leq-1$ & 15,7 & 16,4 & 0,9 \\
P/A & & & \\
Escore $\leq-2$ & 1,8 & 0 & 0,3 \\
Escore $>-2$ e $\leq-1$ & 21 & 4,9 & $0,004^{*}$ \\
A/I & & & \\
Escore $\leq-2$ & 8,8 & 9,8 & 0,58 \\
Escore $>-2$ e $\leq-1$ & 21 & 27,9 & 0,23 \\
\hline
\end{tabular}

GI = grupo pré-intervenção; GII = grupo pós-intervenção

1 Teste do $\chi^{2}$.

Tabela 5

Valores médios e desvios-padrão de hemoglobina ( $\mathrm{g} / \mathrm{dl}$ ) de Gl e GIl, segundo idade, em meses. Projeto Favela, São Paulo, Brasil, 1995-2000.

\begin{tabular}{lrrrrr}
\hline $\begin{array}{l}\text { Faixa etária } \\
\text { (meses) }\end{array}$ & $\mathrm{n}$ & $\begin{array}{r}\text { G I } \\
(\mathrm{X} \pm \mathrm{DP})\end{array}$ & $\mathrm{n}$ & $\begin{array}{c}\text { G II } \\
(\mathrm{X} \pm \mathrm{DP})\end{array}$ & Valor de $\mathbf{p}^{1}$ \\
\hline $12-18$ & 22 & $9,8 \pm 1,3$ & 21 & $10,9 \pm 1,2$ & $0,006^{*}$ \\
$18-26$ & 35 & $10,4 \pm 1,5$ & 36 & $11,1 \pm 1,2$ & $0,048^{*}$ \\
Total & 57 & $10,1 \pm 1,4$ & 57 & $11,0 \pm 1,2$ & $<0,001^{*}$ \\
\hline
\end{tabular}

GI = grupo pré-intervenção; GII = grupo pós-intervenção.

1 Teste $t$ de Student. tanto, o GI exibiu um porcentual significantemente maior de crianças que não inciaram a amamentação. Já no GII, o porcentual de crianças que tiveram aleitamento total acima de 180 dias foi significantemente superior. Ressalta-se que neste último as mães eram mais jovens, inclusive com maior número de adolescentes, fato que poderia conferir maior risco para o desmame precoce.

No país observa-se uma tendência de aumento na prevalência de aleitamento materno. Segundo estudo de Barros \& Victora (1990), durante a década de 80 apenas $6 \%$ das crianças brasileiras eram amamentadas até os dois meses de idade, sendo que $90 \%$ delas iniciaram a amamentação. Já em 1996, segundo a Pesquisa Nacional sobre Demografia e Saúde (BEMFAM, 1997), cerca de $40 \%$ das crianças foram amamentadas até os quatro meses de idade.

Couri et al. (2001) observaram em unidades básicas de saúde da cidade de São Paulo que, embora as mães tivessem bom conhecimento sobre a importância do aleitamento materno, determinadas situações práticas que freqüentemente promovem o desmame não tiveram índices satisfatórios, destacando que apenas o conhecimento não é suficiente para aumentar o período de aleitamento. O suporte dos serviços de saúde para a preparação das mães na superação de dificuldades circunstanciais que ocorrem durante a lactação é fundamental para o estabelecimento da mesma (Garcia-Montrone \& Rose, 1996). Nesse sentido, as ações do Projeto Favela oferecem apoio constante às mães por meio das visitas domiciliares, sobretudo durante o primeiro mês de lactação, período mais critíco para o seu estabelecimento. No entanto, reconhece-se que o aleitamento materno ainda permanece muito abaixo do ideal e exige, portanto, outra abordagem de investigação para que se revelem questões importantes relacionadas à essa prática, permitindo, inclusive, reavaliar as práticas educativas desenvolvidas junto às mães pela equipe do Projeto Favela.

As análises referentes aos indicadores antropométricos indicam um controle sobre os déficits ponderais, uma vez que não houve nenhuma criança do Grupo II com escore $\mathrm{Z}$ abaixo de -2 para os indicadores $\mathrm{P} / \mathrm{I}$ e P/A, persistindo porém $16,4 \%$ de crianças em risco nutricional $(Z<-1)$ para o primeiro. Tal achado estaria justificado uma vez que o indicador $\mathrm{P} / \mathrm{I}$ reflete em parte a adequação da estatura, e para esta, os déficits encontrados foram elevados no GII ainda que sem diferença significante entre os dois grupos. Reichenheim \& Harpham (1990) afirmam que esse padrão é compatível 
com o quadro de taxas de mortalidade infantil intermediárias e, nesse contexto, também são menores os agravos devido a carências extremas de alimentos e infecções graves. Segundo o mesmo autor, essa situação permite a adaptação das crianças, reduzindo seu crescimento linear, sem déficits importantes de peso.

Assis \& Barreto (2000) confirmam que o padrão da distribuição dos déficit de P/I e A/I, tradicionalmente observado até recentemente no Brasil, é caracterizado por exibir altas taxas de inadequação de altura em relação à idade, seguidas por taxas menores de inadequação do peso em relação à idade. Como exemplo, temse os dados do PNSN (1989) para o Nordeste urbano, onde a prevalência de déficits de A/I era de $23,9 \%$ e de $\mathrm{P} / \mathrm{I}$ era de $10,3 \%$.

Em São Paulo, o déficit estatural para o tercil mais pobre da população está em 3,6\% e o déficit ponderal em $0,6 \%$, sugerindo o virtual desaparecimento desse último (Monteiro \& Conde, 2000). No entanto, Mesquita et al. (2001), em estudo com crianças de outras favelas da Zona Sul da cidade, encontrou prevalência de DEP, segundo o indicador P/I de $26 \%$, revelando que os déficits ponderais ainda representam um problema importante quando se focalizam populações vivendo em condições de pobreza, como é o caso das favelas. Nesse sentido, o resultado deste estudo para os indicadores relacionados ao peso são extremamente favoráveis.

Segundo Victora (1992), os determinantes do ganho de peso e do crescimento linear possuem alguns componentes distintos. $\mathrm{O}$ déficit de estatura está associado de forma predominante às condições sócio-econômicas, como trabalho e escolaridade dos pais e, possivelmente, à deficiência crônica de micronutrientes; de outro modo, o déficit ponderal reflete em maior grau as condições vividas no momento, como a ocorrência de doenças. Nesse sentido, Taddei (2000) destaca que as desigualdades que evidenciam a vulnerabilidade socioeconômica são mais extensas que a conferida pelo risco biológico de ser menor de 24 meses. Dessa forma, o setor saúde tem limitado seu espaço de ação, sendo que a melhoria do crescimento linear da criança, por representar a exposição crônica às condições sociais desfavoráveis, embora não exclua as ações do setor saúde, passa invariavelmente por progressos na distribuição de renda (Angstrom \& Anjos, 1999).

Alguns fatores que compõem o modelo téorico de determinação da DEP não estão contemplados neste estudo, limitando, desse modo, as inferências sobre o papel da intervenção na redução dos déficits antropométricos. A ren- da per capita, sabidamente o determindante distal mais importante para a condição nutricional da criança, não foi analisada. A obtenção dessa informação é díficil em favelas, posto que grande parte da população não possui vínculo empregatício e, muitas vezes, a renda advém do trabalho informal e temporário (Schmitz, 1999). No entanto, considerou-se a escolaridade materna como um proxy dessa condição (Mueller \& Parcel, 1981), e de acordo com esse indicador não houve diferença entre os grupos. Em relação ao abastecimento de água da rede pública, rede de esgoto e coleta de lixo, no ano do diagnóstico do GI, quase $100 \%$ das casas contavam com esses serviços, não se esperando maiores avanços, entretanto essa análise não foi realizada. Outra questão diz respeito à própria tendência da desnutrição no Município de São Paulo, claramente de queda, também dificultando atribuir, com precisão, a melhoria dos indicadores antropométricos somente à intervenção.

A prevalência da anemia, ao contrário da DEP, tem revelado tendência ascendente no país, inclusive na Região Sudeste. No grupo pósintervenção (GII), observou-se redução significante na prevalência, apesar de ainda se manter em um patamar bastante elevado (50\%). Dados de outras favelas, da mesma cidade, para crianças menores de 24 meses, revelaram prevalência de 66,7\% (Sigulem et al., 2001), valor próximo ao observado por Silva et al. (2001), também em menores de 24 meses, na cidade de Viçosa, Minas Gerais (65\%). Ribeiro (2001), em diagnóstico realizado em 334 crianças de creches municipais de São Paulo, que atendem crianças de baixa renda, apontou prevalência de $69 \%$ em crianças de 13 a 24 meses.

A anemia de maior gravidade sofreu redução ainda mais importante, passando de $33 \%$ para $7 \%$. Prevalências superiores foram encontadas por Silva et al. (2001), em Viçosa e Ribeiro (2001), em São Paulo, com 50\% e 32,1\%, respectivamente.

O valor médio de hemoglobina também revelou-se significantemente maior no GII (11,0 $\pm 1,2 \mathrm{~g} / \mathrm{dl}$ ), valor esse que também se situa acima dos valores observados por Sigulem et al. (2001) e Ribeiro (2001), em populações de baixa renda da cidade de São Paulo, que encontraram concentração média de 10,2 \pm 1,7 e 9,6 \pm $1,1 \mathrm{~g} / \mathrm{dl}$, respectivamente. Esses resultados são mais consistentes quando se observa a tendência da anemia na cidade de São Paulo. Essa tendência é evidenciada pelos estudos de Sigulem et al. (1978) e Monteiro et al. (2000b), com amostras probabilísticas, quando em 1974 a prevalência era de 35\% passando, em 1996, pa- 
ra $67.6 \%$ na faixa etária de 6 a 24 meses. O valor médio da hemoglobina também reduziu entre a década de 80 e 90, passando de 10,7 \pm 2,0 para $10,3 \pm 1,7 \mathrm{~g} / \mathrm{dl}$.

Embora a prevalência de anemia seja maior nos extratos mais pobres da população, a renda familar parece não ser o fator determinante mais importante para a sua ocorrência (Assis et al., 1997; Sigulem et al., 1978). Durante os dois primeiros anos de vida, o acelerado processo de crescimento, o abandono precoce do aleitamento materno e a dieta, predominantemente láctea, são fatores importantes na etiologia da anemia ferropriva (Sigulem et al., 1978). No presente estudo, a dieta das crianças não foi analisada, mas outros estudos realizados na mesma favela em diferentes momentos apontam, claramente, para a baixa adequação de ferro na dieta (Devincenzi, 1999; Schmitz, 1999), fato também observado na cidade de São Paulo por Monteiro et al. (2000b).

A redução da anemia ferropriva, atualmente, representa o maior desafio entre as carências nutricionais no Brasil. Seu controle parece estar ainda distante, apesar de diversos estudos de intervenção que vêm se realizando desde a década de 90. Torres et al. (1994), com esse objetivo, estudaram a intervenção realizada em crianças de 6 a 36 meses, em unidades básicas de saúde de São Paulo. Ao final, os autores concluiram que apenas $30 \%$ das mães ofereceram o medicamento regularmente, sendo a adesão ao tratamento, o grande fator restritivo para o sucesso do programa. Tendo em vista essa perspectiva, a rotina de visita domiciliar, adotada pelo Projeto Favela, pode contribuir para maior adesão ao tratamento e/ou profilaxia medicamentosa.

Childs et al. (1997), ao avaliarem intervenções educativas para crianças no primeiro ano de vida, voltadas para a prevenção da anemia, não encontraram diferenças entre o grupo controle e de intervenção. Os autores ressaltaram que programa educativo, com o objetivo de reduzir a incidência de anemia, deve ser acompanhado de prevenção secundária, com diagnóstico e tratamento da população em risco. Deve ser ressaltado que cada grupo populacional, em virtude de suas diferentes características e condições fisiológicas, necessitam de ações diferenciadas para o controle da anemia e que as crianças até 24 meses privilegiam-se com essa combinação. Nesse sentido, o Projeto Favela utiliza-se de ações educativas como também de ações de diagnóstico, tratamento e profilaxia, tendo em vista as características particulares do grupo pré-escolar.

Os resultados deste estudo, revelam o potencial que a incorporação de ações para prevenção e controle de carências nutricionais na atenção primária à saúde tem na melhoria da situação nutricional na infância. A validade para outros grupos, em princípio, se limitaria a populações carentes de grandes conglomerados urbanos devido à própria operacionalização da proposta de intervenção. Entretanto, destacase que as atividades implementadas utilizam tecnologias simplificadas, de fácil reprodutibilidade e de efetiva utilidade.

\section{Referências}

ANGSTROM, E. M. \& ANJOS, L. A., 1999. Déficit estatural nas crianças brasileiras: Relação com condições socioambientais e estado nutricional materno. Cadernos de Saúde Pública, 15:559-567.

ASSIS, A. M. O. \& BARRETO, M. L., 2000. Condições de Vida, Saúde e Nutrição na Infância em Salvador Salvador: Instituto de Saúde Coletiva, Universidade Federal da Bahia.

ASSIS, A. M. O.; SANTOS, L. M. P.; MARTINS, M. C.; ARAÚJO, M. P. N.; AMORIM, D. Q.; MORRIS, S. S. \& BARRETO, M. L., 1997. Distribuição da anemia em pré-escolares do semi-árido da Bahia. Cadernos de Saúde Pública, 13:247-243. 
BARROS, F. C. \& VICTORA, C. G., 1990. Breastfeeding and Diarrhea in Brazilian Children. Demographic and Health Surveys Further Analyses Series 3. New York: Population Council.

BEMFAM (Sociedade Civil Bem-Estar Familiar no Brasil), 1997. Programas de Pesquisas de Demografia e Saúde (DHS); Brasil, Pesquisa Nacional sobre Demografia e Saúde. Rio de Janeiro: BEMFAM.

CAETANO, L. C., 1992. Aleitamento Materno: Fatores que Contribuem para a sua Prática. Dissertação de Mestrado, São Paulo: Escola Paulista de Medicina, Universidade Federal de São Paulo.

CHILDS, F; AUKETT, A.; DARBYSHIRE, P.; ILETT, S. \& LIVERA, L. N., 1997. Dietary education and iron deficiency anemia in the inner city. Archives of Disease in Childhood, 76:144-152.

COURI, A.; BACHILLER, C. \& GOULART, R. M. M., 2001. Conhecimentos maternos em relação ao aleitamento natural. In: III World Congress of Pediatric Nutrition, Resumos, p. 41, São Paulo: Sociedade Brasileira de Pediatria.

DEVINCENZI, M. U., 1999. Anemia Ferropriva na Primeira Infância: Intervenção com Atenção Primaria à Saúde. Dissertação de Mestrado, São Paulo: Escola Paulista de Medicina, Universidade Federal de São Paulo.

FERNANDES, B. S.; JERÔNIMO, M. L. \& LEONE, C., 1996. Características familiares e cuidados e condições de saúde das crianças: Seu papel no risco de desnutrição protéico-calórica. Pediatria, 18: 65-74.

GARCIA-MONTRONE, V. \& ROSE, J. C., 1996. Uma experiência educacional de incentivo ao aleitamento materno e estimulação do bebê, para mães de nível sócio-econômico baixo: Estudo preliminar. Cadernos de Saúde Pública, 12:61-68.

JELLIFFE, D. B., 1968. The Assessment of the Nutritional Status of the Comunity. Geneva: World Health Organization.

KIRKWOOD, B. R.; COUSENS, S. N.; VICTORA, C. G. \& ZOYSA, I., 1997. Issues in the design and interpretation of studies to evaluate the impact of community-based interventions. Tropical Medicine and International Health, 2:1022-1029.

KLEIN, R. E.; READ, M. S.; RIECKEN, H. N.; BROWN Jr., J. A.; PRADELLA, A. \& DAZA, C. H., 1982. Evaluación del Impacto de los Programas de Nutrición y de Salud. Washington, D.C.: Organización Panamericana de la Salud.

MESQUITA, K.; SILVA, A. C.; KIRSCH, A.; MARTINS, B.; NASSIF, P.; KIRSCH, M.; IAMAMURA, M.; ZOLLNER, A. C.; JULIANO, Y.; NOVO, N. F.; CURY, M. C. \& COLOMBO, P., 2001. Estado nutricional de crianças atendidas no Centro de Saúde Escola. In: III World Congress of Pediatric Nutrition, Resumos, p. 37, São Paulo: Sociedade Brasileira de Pediatria.

MONTEIRO, C. A. \& CONDE, W. L., 2000. Tendência secular da desnutrição e da obesidade na infância na Cidade de São Paulo (1974-1996). Revista de Saúde Pública, 34(Sup. 6):52-61.

MONTEIRO, C. A. \& NAZÁRIO, C. L., 2000. Evolução de condicionantes ambientais da saúde na infância na Cidade de São Paulo (1984-1996). Revista de Saúde Pública, 34(Sup. 6):13-18.

MONTEIRO, C. A. \& SZARFARC, S. C., 1987. Estudo das condições das crianças no Município de São Paulo, SP (Brasil), 1984-1985. V - Anemia. Revista de Saúde Pública, 21:255-260.

MONTEIRO, C. A.; BENÍCIO, M. H. \& ORTIZ, L., 2000a. Tendência secular do peso ao nascer na Cidade de São Paulo (1974-1996). Revista de Saúde Pública, 34(Sup. 6):26-40.

MONTEIRO, C. A.; SZARFARC, S. C. \& MONDINI, L., 2000b. Tendência secular da anemia na infância na Cidade de São Paulo. Revista de Saúde Pública, 34(Sup. 6):62-72.

MUELLER, C. W. \& PARCEL, T. I., 1981. Measures of socioeconomic status: Alternatives and recommendations. Child Development, 52:13-30.

NOBREGA, F. J.; VÍTOLO, M. R.; BRASIL, A. L. \& LOPES, F. A., 1991. Condição nutricional de mães e filhos: Relação com o peso ao nascimento, variáveis maternas e socioeconômicas. Jornal de Pediatria, 67:288-296.

OMS (Organización Mundial de la Salud), 1968. Anemias Nutricionales: Informes de um Grupo Científico. Série de Informes Técnicos 405. Geneva: OMS.

OMS (Organización Mundial de la Salud), 1989. Urbanización y sus Repercusiones en la Salud Infantil-Posibilidades de Acción. Geneva: OMS.

PRIORE, S. E.; SOUZA, M. H. N.; SOLYMOS, G. M. B.; FRANCESCHINI, S. C. C. \& SIGULEM, D. M., 1994. Projeto Favela: Um modelo de atenção à saúde. Saúde Para Todos, 4:43-45.

REICHENHEIM, M. E. \& HARPHAM, T., 1990. Perfil intracomunitário da deficiência nutricional: Estudo de crianças abaixo de 5 anos numa comunidade de baixa renda do Rio de Janeiro (Brasil). Revista de Saúde Pública, 24:69-79.

RIBEIRO, L. C., 2001. Anemia Ferropriva na Primeira Infância: Controle e Prevenção com Doses Intermitentes de Ferro Quelato Glicinato. Dissertação de Mestrado, São Paulo: Escola Paulista de Medicina, Universidade Federal de São Paulo.

ROSENBILIT, J.; ABREU, C. R.; SZTERLING, L. N.; KUTNER, J. M.; HAMERSCHLAK, N.; FRUTUOSO, P; PAIVA, T. R. S. S. \& FERREIRA Jr., O. C., 1999. Evaluation of three methods for hemoglobin measurement in a blood donor setting. Revista Paulista de Medicina, 117:108-112.

ROSENFIELD, L. G. M. \& SIGULEM, D. M., 1988. Recomendações profiláticas e terapêuticas na anemia. Boletim da Sociedade Brasileira de Hematologia e Hemoterapia, 10:177-180.

SANTOS, M. A., 1982. Urbanização Desigual: A Especificidade do Fenômeno Urbano em Países Subdesenvolvidos. Rio de Janeiro: Editora Vozes.

SCHMITZ, B. A. S., 1999. Avaliação de um Programa de Atenção Primária à Saúde: Impacto sobre o Estado Nutricional. Tese de Doutorado, São Paulo: Escola Paulista de Medicina, Universidade Federal de São Paulo.

SIGULEM, D. M.; TUDISCO, E. S.; GOLDEMBERG, P.; ATHAIDE, M. M. \& VAISMAN, E., 1978. Anemia ferropriva em crianças do Município de São Paulo. Revista de Saúde Pública, 12:168-179.

SIGULEM, D. M., 1981. Condições de Saúde e Nutrição em Crianças do Município de São Paulo. Tese de Doutorado, São Paulo: Escola Paulista de Medicina, Universidade Federal de São Paulo. 
SIGULEM, D. M.; SOUZA, P. C.; ZOLLNER, A. C. R.; NOVO, N. F.; JULIANO, Y. \& CURY, M. C. F. S., 2001. Anemia carencial em lactentes e pré-escolares atendidos no Centro de Saúde Escola. In: III World Congress of Pediatric Nutrition, Resumos, p. 37, São Paulo: Sociedade Brasileira de Pediatria.

SILVA, D.; ROCHA, D.; NETO, M.; FONSECA, M.; MIRANDA, A.; FRANCHESCHINI, S.; FRANCHESCHINI, S. C. C.; PRIORE, S. E.; EUCLYDES, M. P.; RIBEIRO, S. M. R. \& ARAUJO, R. M. A., 2001. Caracterização da anemia ferropriva em crianças de dois grupamentos etários do Município de Viçosa. In: III World Congress of Pediatric Nutrition, Resumos, p. 29, São Paulo: Sociedade Brasileira de Pediatria.

TADDEI, C. A. A. C., 2000. Desvios Nutricionais em Menores de 5 anos: Evidências dos Inquéritos $\mathrm{Na}$ cionais. Tese de Livre-Docência, São Paulo: Escola Paulista de Medicina, Universidade Federal de São Paulo.

TORRES, M. A. A.; SATO, K. \& QUEIROZ, S. S., 1994. Anemia em crianças menores de dois anos atendidas nas Unidades Básica de Saúde no Estado de São Paulo. Revista de Saúde Pública, 28:290-294.

UNICEF (Fundo das Nações Unidas para a Infância), 1979. Cuidados Primários de Saúde - Relatório da Conferência Internacional sobre Cuidados Primários de Saúde-Alma-Ata, 1978. Brasília: UNICEF.

UNICEF (Fundo das Nações Unidas para a Infância),
1998. Situação Mundial da Infância - A Nutrição em Foco. Brasília: UNICEF.

VICTORA, C. G., 1992. The association between wasting and stunting: An international perspective. Journal of Nutrition, 122:1105-1110.

WHO (World Health Organization), 1977. WHO: Recommended definitions, terminology and format for statistical tables related to the perinatal period and use of a new certificate for cause of perinatal deaths. Acta Obstetricia et Gynecologica Scandinavica, 56:247-253.

WHO (World Health Organization), 1991. Indicators for Assessing Breastfeeding Practices. Geneva: WHO.

WHO (World Health Organization), 1995. Physical Status: The Use and Interpretation of Antropometric. Geneva: WHO.

YUNES, R. F. \& MONTEIRO, C. A., 1995. Razões para a Melhoria do Estado Nutricional das Crianças Brasileiras nas Décadas de 70 e 80. São Paulo: Núcleo de Pesquisas Epidemiológicas em Nutrição e Saúde, Universidade de São Paulo.

Recebido em 22 de fevereiro de 2002

Versão final reapresentada em 7 de outubro de 2002

Aprovado em 27 de novembro de 2002 